\title{
Coaching by non-drug prescribing health professionals reduced total cholesterol concentrations in coronary heart disease
}

Vale MJ, Jelinek MV, Best JD, et al. Coaching patients On Achieving Cardiovascular Health (COACH): a multicenter randomized trial in patients with coronary heart disease. Arch Intern Med 2003;163:2775-83.

\section{$Q$ In patients with coronary heart disease (CHD), does a 6 month programme of coaching by non-drug prescribing nurses and dietitians reduce total cholesterol (TC) concentrations?}

\section{METHODS}

Design: randomised controlled trial (Coaching patients On
Achieving Cardiovascular Health [COACH]).
Allocation: concealed.
Sellow up period: 6 months.
Pospitals in Melbourne, Australia.
Patients: 792 patients (mean age 59 y, $77 \%$ men) who were
admitted to hospital for coronary artery bypass graft surgery;
percutaneous coronary intervention; acute myocardial infarction
or unstable angina and discharged on medical therapy; or
coronary angiography with planned elective revascularisation.
Exclusion criteria: inaccessibility by telephone, inability to speak
or read English or travel to hospital for follow up visits, no fasting
blood sample taken within 24 hours of admission, participation in
another lipid study, or too ill to be interviewed in hospital.
Intervention: COACH programme (n=398) or usual care
(n=394). Coaches were 2 dietitians and 4 nurses who
telephoned patients within 2 weeks of randomisation and another
4 times during follow up. Coaches encouraged patients to visit
their family physicians to obtain measurements of their risk
factors and negotiated a plan of action to achieve target lipid
concentrations (TC $<4.0$ mmol//) and reduce other coronary risk
factors. Written reports of each coaching session were sent to
patients after each call.
Outcomes: 6 month change in fasting serum TC concentrations
from baseline. Secondary outcomes included high and low
density lipoprotein cholesterol concentrations, blood pressure,
body weight and body mass index (BMI), and dietary fat intake.
Patient follow up: $86 \%$ (intention to treat analysis).
For correspondence: Dr M J Vale, St Vincent's Hospital Melbourne, Fitzroy, Victoria, Australia. margarite.vale@svhm.org.au

Sources of funding: Victorian Health Promotion Foundation and Merck Sharp and Dohme.

\section{MAIN RESULTS}

Patients in the $\mathrm{COACH}$ group had greater decreases in TC concentrations than patients in the usual care group (table). The $\mathrm{COACH}$ programme also reduced low density lipoprotein cholesterol concentrations, weight, BMI, dietary fat intake, and anxiety more than usual care. Blood pressure increased in both groups but to a lesser extent in the COACH group (table).

\section{CONCLUSION}

In patients with coronary heart disease, telephone coaching by nondrug prescribing nurses and dietitians reduced total cholesterol concentrations and other coronary risk factors.

A modified version of this abstract appears in Evidence-Based Medicine.

\section{Commentary}

R esearch on the effectiveness of multicomponent disease management programmes for improving coronary risk profiles is slowly emerging. ${ }^{2}$ However, evidence regarding the effectiveness of individual components of these programmes, specifically non-prescribing nurse led interventions, is limited and conflicting. ${ }^{3}$ The trial by Vale et al rigorously examined the effectiveness of nurse or dietitian coaching on achieving cardiac risk factor targets, particularly serum TC concentrations.

Patients from 6 Australian teaching hospitals were contacted by health workers who had received training on the $\mathrm{COACH}$ programme. The programme assists patients to aggressively pursue improvements in cardiac risk factors. It reflects common principles of self care: processes are initiated by the individual or in collaboration with a healthcare professional, and involve active client participation in health related decisions and actions. ${ }^{4}$ The programme focuses on the development of self management skills beyond mere understanding of treatment targets to include proactive communication with physicians and development of action plans.

Nurses with or without prescribing authority could incorporate coaching strategies into their care and follow up patients with modifiable risks. Nurses have an important role in supporting and encouraging patients' self management of chronic illness, and the nature of nursing is well suited to coaching, a function that may enhance the effectiveness of more passive educational strategies.

Joan E Tranmer, RN, PhD Kingston General Hospital and Queen's University Kingston, Ontario, Canada

1 McAlister FA, Lawson FM, Teo KK, et al. BMJ 2001;323:957-62.

2 Rich MW. J Card Fail 1999;5:64-75.

3 Naylor MD, Brooten D, Campbell R, et al. JAMA 1999;281:613-20

4 Sidani S. Can J Nurs Leadersh 2003;16:63-5.

Coaching in cardiovascular health $v$ usual care for coronary heart disease*

\begin{tabular}{lccc}
\hline Outcomes at 6 months & COACH & Usual care & Difference in mean change (95\% Cl) \\
\hline Total cholesterol (mmol/l) & -0.54 & -0.18 & $0.36(0.20$ to 0.52$)$ \\
BMI $\left(\mathrm{kg} / \mathrm{m}^{2}\right.$ ) & -0.5 & -0.1 & $0.4(0.1$ to 0.5$)$ \\
Total fat $(\mathrm{g})$ & -15.3 & -10.5 & $4.8(0.3$ to 9.3$)$ \\
Systolic BP $(\mathrm{mm} \mathrm{Hg})$ & +0.1 & +4.5 & $4.4(1.8$ to 7.0$)$ \\
Diastolic BP (mm Hg) & +0.4 & +2.8 & $2.4(0.7$ to 4.0$)$ \\
\hline \multirow{2}{*}{$\mathrm{BMI}=$ body mass index; BP = blood pressure. Values are mean change from baseline. Cls provided by author. }
\end{tabular}

